\title{
The Evaluation of Predictive Learners: Some Theoretical and Empirical Results
}

\author{
Kevin B. Korb, Lucas R. Hope, and Michelle J. Hughes \\ School of Computer Science and Software Engineering \\ Monash University, VIC 3800, Australia, \\ $\{$ korb, lhope,mjh\}@csse.monash.edu.au,
}

\begin{abstract}
With the growth of interest in data mining, there has been increasing interest in applying machine learning algorithms to real-world problems. This raises the question of how to evaluate the performance of machine learning algorithms. The standard procedure performs random sampling of predictive accuracy until a statistically significant difference arises between competing algorithms. That procedure fails to take into account the calibration of predictions. An alternative procedure uses an information reward measure (from I.J. Good) which is sensitive both to domain knowledge (predictive accuracy) and calibration. We analyze this measure, relating it to Kullback-Leibler distance. We also apply it to five well-known machine learning algorithms across a variety of problems, demonstrating some variations in their assessments using accuracy vs. information reward. We also look experimentally at information reward as a function of calibration and accuracy.
\end{abstract}

Keywords: Evaluation, information reward, predictive accuracy, scoring rules, machine learning, Kullback-Leibler distance.

\section{Introduction}

With the growing accessibility of computational power and accumulation of data in large databases, machine learning is rapidly becoming a central concern for both academia and industry, as the growing interest in applying machine learning to data mining demonstrates. A difficult and important meta-question has yet to be answered satisfactorily: how do we decide whether one machine learning algorithm is superior to another? The standard practice has been to take some collection of samples in the domain of interest, select some random subset as training cases to which the machine learning algorithm is applied and run the representation thus learned on the test cases remaining, using the resultant estimate of predictive accuracy as the index of success for the algorithm. This is not a bad practice, particularly once the standards of statistical inference were attended to and, especially, sample sizes were increased, allowing for the calculation of a sensible estimate of the standard deviation in predictive accuracy.

Nevertheless, there are a number of likely objections to this use of predictive accuracy for assessment. Orthodox statistical practices, and in particular the 
significance testing which this practice relies upon, are beset with foundational difficulties. For example, it is easy to find circumstances in which the finding of a significant result - leading to the rejection of the null hypothesis, per Jerzy Neyman-results in an increased posterior probability that the null hypothesis is true. Or, again, one can derive 95\% classical confidence interval estimates (equivalent to significance tests under a transformation) which have a probability of zero of capturing the population parameter of interest [Leslie, 1998]. These sorts of objections are commonly advanced by Bayesians and, if taken seriously, should lead to reform in the selection and use of statistical inference techniques.

But there is an even more telling, even compelling, objection to the standard practice of assessment by predictive accuracy. Predictive accuracy entirely disregards the confidence of the prediction. In binomial classification, for example, a prediction of a mushroom's edibility with a probability of 0.51 counts exactly the same as a prediction of edibility with a probability of 1.0. Now, if we were confronted with the first prediction, we might rationally hesitate to consume such a mushroom. The predictive accuracy measurement does not hesitate. According to standard evaluation practice in machine learning and data mining every prediction is as good as every other. Any business, or animal, which behaved this way would have a very short life span.

The typical Bayesian approach to prediction is to calculate the expected value of a classification, which explicitly incorporates the probability given to a class. Indeed, Bayesianism typically goes further than to hesitate concerning a predicted class, it even hesitates about the model used to classify; that is, the ideal Bayesian approach is to compute a posterior distribution over the entire model space, and then to combine the individual predictions of the models, weighting them according to their posteriors. This mixed prediction will typically provide better predictions than those of any of the individual models, including that one with the highest posterior probability. This is the hard-core Bayesian method, as advocated, for example, by Richard Jeffrey [Jeffrey, 1983. But there are many situations where this ideal method is unavailable. It is in many (or most) inductive environments computationally intractable, requiring joint probability distributions over all variables in the model space, extending to any hypotheses in other domains that may be found to be relevant. More typical of actual scientific practice is that we come to believe a particular scientific theory, which is then applied individually to make predictions or to guide interventions in related domains. So, in machine learning, a possible compromise approach to evaluating machine learners would be to take the posterior probabilities of individual hypotheses put forward by the machine learners as guidance as to which algorithm is the best. Of course, if one of the machine algorithms under consideration is Bayesian, then this Bayesian assessment will always favor it. So, it would be useful to have some evaluation metric for the predictive success of learning algorithms which does not simply assume Bayesianism, as Bayesian principles are in part what is at stake in the methodology of machine learning. 
A good starting point for such an alternative is the concept of KullbackLeibler distance. This measures the "distance" between two probability distributions $p(\cdot)$ and $q(\cdot)$, according to the formula Cover and Thomas, 1991]:

$$
K L(p, q)=\sum_{x \in X} p(x) \log \frac{p(x)}{q(x)}=E_{p} \log \frac{p(x)}{q(x)}
$$

This measure records the distance from $p$ to $q$, from the "point of view" of $p$. When $p$ and $q$ are identical, the KL distance is zero; as they diverge, the KL distance may tend towards infinity. This is the right measure when there is a preferred "point of view" — when the true probability distribution is known, and if that is $p$ in equation (11), then KL distance reports how close a learned distribution $q$ is to the generating distribution. This provides a performance measure for machine learning algorithms that increases monotonically as the learned distribution diverges from the truth. Also, being based strictly upon the output distribution of a machine learning algorithm, it is invariant to the internal properties of the algorithm itself, including the complexity of the representations used, as is appropriate since such a performance measure is not attempting to assess the probability of those representations directly. The drawback to KL distance is simply that when assessing algorithms in the real world, the true model is necessarily unknown. We would like a performance measure with similar properties, but which does not depend on prior knowledge of the truth.

\section{Knowledge and Meta-knowledge}

There are two fundamental ingredients to predictive success: knowledge of the system whose behavior is being predicted and knowledge of the limits of that knowledge. With the former comes a greater ability to predict target states; with the latter comes an improved ability to assess the probability that those predictions are in error. These two are not in a trade-off relationship: they can be jointly maximized. Given fixed domain knowledge, it is a theorem that to maximize betting reward, one must calibrate the odds - over- or under-estimating them guarantees (in the long run) suboptimal performance (Cover and Thomas, 1991, chapter 6) 1 And, as Ramsey, 1931. argued effectively, betting is a pertinent metaphor for every kind of decision making under uncertainty, including the prediction tasks to which machine learners might be put.

Orthodox machine learning assessment of accuracy for classification tasks simply sums up all the test cases classified correctly and divides by the total number of cases. Clearly, using such a metric, it does not pay to withhold classification, for that guarantees a failure to classify correctly, which is all that is being rewarded here. In general, it does not pay to express any doubts whatsoever about one's classifications: given that a probability of a target class is greater than 0.5 (in the binomial case) one should always act as if the probability is 1 .

\footnotetext{
${ }^{1}$ In philosophy this is recognized in David Lewis's "Principal Principle", which asserts that one's subjective probability for an event, conditioned upon the knowledge of a physical probability of that event, should equal the latter [Lewis, 1980].
} 
As noted above, such behavior applied to ordinary tasks of an agent trying to get around in the world would lead to rapid extinction. This metric ignores the costs of misclassification and the rewards of correct classification, as some AI researchers have begun pointing out (e.g., Turney, 1995 Provost et al., 1998]; also see the earlier Pearl, 1978]). A more appropriate metric, therefore, would be a sample estimate of the expected value of the classification, that is, something like:

$$
\frac{\text { reward } \times s+\text { penalty } \times(n-s)}{n}
$$

where $n$ is the number of test items, $s$ the number of correct predictions and reward and penalty the rewards for correct and incorrect predictions respectively. Such a metric (or more complex relatives) we think to be correct, whenever applicable. Unfortunately, they are often not applicable. In general, in science one cannot well anticipate the uses to which scientific inferences will be put, and so one cannot know the values associated with correct or incorrect conclusions. This holds also for evaluating machine learning and data mining techniques: the number and range of applications is entirely open ended, and so too therefore are the rewards and penalties. We need a metric which somehow produces results consistent with "normal" values for penalties and rewards.

\section{The Information Reward}

We suggest a proper such metric for assessing machine learning algorithms is simply how well those algorithms would perform in guiding the betting behavior of agents located in the domain or world of interest. In other words, if the learning algorithm can consistently beat its competitors when betting on domain events, then it is superior to its competitors (within that domain). This is an ability which the orthodox measure of predictive accuracy fails to assess 2

Lacking an exact characterization of costs and rewards available for incorrect and correct classifications, we use the information-theoretic reward Good, 1952 Dowe et al., 1996]: reward $=\sum_{i}\left[1+\log _{2} P\left(X_{i}\right)\right]$ where $i$ indexes the test cases, $X_{i}$ is the actual class of the $i$ th test case and $P\left(X_{i}\right)$ is the probability of that event asserted by the learner 3 This function demonstrably has the two properties we require of our metric. First, given perfect calibration, the reward is monotonic to domain knowledge, with the limit of 1 "bit" for each classification known with certainty and 0 bits for complete ignorance in the binomial classification task (i.e., $P\left(X_{i}\right)=0.5$ ). This reward is one minus the length of a message conveying the actual event in a language efficient for agents with

2 Kononenko and Bratko, 1991 also criticize the use of predictive accuracy as a comparative scoring function, and provide an information based scoring function which explicitly takes prior probabilities of classes into account. Their work will be dealt with in a more comprehensive paper.

${ }^{3}$ For multinomial classification we used the above equation for successful classifications; for misclassifications we treated all the non-selected classes as a contrast class (i.e., grouping them into one class) - i.e., $1+\log _{2}\left(1-P\left(X_{i}\right)\right.$ ). 
the learner's beliefs (i.e., a language that minimizes entropy for them) 4 Second, given fixed domain knowledge, it is maximized by perfect calibration. Taking the binomial case for simplicity, let $c$ be the physical probability (chance) that some test case is in the target class and $p$ the probability estimated by the machine learner. Then the expected reward function is:

$$
\left[1+\log _{2} p\right] c+\left[1+\log _{2}(1-p)\right](1-c)
$$

To find the maximum we take the derivative with respect to $p$ and set it to zero:

$$
\begin{aligned}
0 & =\frac{d}{d p}\left(\left[1+\log _{2} p\right] c+\left[1+\log _{2}(1-p)\right](1-c)\right) \\
& =\frac{c}{p}+\frac{1-c}{1-p} \frac{d}{d p}(1-p) \\
& =\frac{c}{p}-\frac{1-c}{1-p} \\
c & =p
\end{aligned}
$$

which last equation reports that perfect calibration maximizes reward.

\section{A Calibration Measure}

The information reward measures domain knowledge and meta-knowledge (calibration) jointly. It would be useful to be able to tease these apart: if we can measure calibration directly, then by comparison with the information reward we can measure domain knowledge indirectly; also, we can use calibration measures to improve performance by recalibrating our prediction probabilities. Calibration has been measured by cognitive psychologists investigating how human cognition deviates from the ideal [Lichtenstein et al., 1977]. The basic idea is to measure how much the probability estimate of the agent deviates from the frequency of truth of events estimated at each particular level. Thus, if the agent gets right $70 \%$ of events that the agent accords a probability of 0.9 , then the agent is overconfident - at least, of events which are given that probability (such overconfidence, by the way, is typical of non-expert humans Lichtenstein et al., 1977). A measure of miscalibration suggested by this is:

$$
\text { miscalibration }=\sqrt{\sum_{i \in I} \sum_{j \in J} \frac{\left(\frac{\sum_{k} f_{i k}}{n_{i}}-p_{i j}\right)^{2}}{n_{i}-1}}
$$

where $I$ partitions the range of probabilistic predictions from 0 to 1 , so we are summing over the partition cells; $J$ and $K$ index the predictions within each

\footnotetext{
${ }^{4}$ Note that such a message is infinite if it is attempting to communicate an event that has been deemed to be impossible, that is, to have probability zero.
} 
partition cell.5 What we sum is the average of the squared deviations of the prediction probabilities $\left(p_{i j}\right)$ from the average frequency of truth within each cell (so $f_{i j}$ is 1 or 0 depending upon whether that particular prediction was correct or not) 6

\section{$5 \quad$ Results}

For this study we tested five well-known machine learning algorithms, using the same datasets employed by Holte, 1993:

- C4.5 Quinlan, 1993: This learns pruned classification trees; the leaves have associated probability distributions over classes.

- MML classification trees Wallace and Patrick, 1993|: This uses MML (Minimum Message Length Wallace and Boulton, 1968) to learn classification trees, with probabilities.

- MML classification graphs Oliver, 1993]: This uses MML to learn classification graphs, with branches that may split or join (making disjunctive concepts easier to learn); leaves have associated classification probabilities.

- Holte's decision stumps Holte, 1993: Robert Holte demonstrated that classification trees with only a single internal node (i.e., with classification based only on a single attribute) can achieve predictive accuracies comparable over many data sets to those of C4.5, which usually learns far more complex representations.

- Naive Bayes: Instead of splitting on a single attribute these simple models split on class membership, with the leaves representing the different available attributes. The models are "naive" because they assume that the attributes, given knowledge of class membership, are independent of each other. Observed attribute values are filled in and a simple Bayesian net propagation provides a posterior probability of class membership. We implemented the algorithm as described in Mitchell, 1997,7

Each algorithm was applied to each dataset, with two-thirds of the data randomly allocated as training data and one-third for testing. Each such test was repeated 25 times to obtain the $95 \%$ confidence intervals shown in Tables 11. 2 and [3.

In assessing the information reward for each algorithm we require a probability associated with each predicted class. In the case of the MML algorithms, predicting probabilities is an integral aspect of their operation. For C4.5, leaf nodes have probabilities associated with classes internally (called "class weights"); we

${ }^{5}$ In our experimental work below we divided the range of probabilities into cells so that each cell was guaranteed to contain at least 10 sample points and as few as possible above that number.

6 This measure does not distinguish between over- and under-confidence, but can easily be adjusted to do so.

7 Our results for Naive Bayes (Table1) are comparable with other experimental reports (e.g., Domingos and Pazzani 1996]). 
modified the source code to externalize those probabilities. For Naive Bayes and decision stumps, we used the frequency associated with the predicted class amongst training set items classified to the appropriate node. Since the information reward can penalize wrong predictions without limit - for example, probabilities of 0 and 1 correspond to offering infinite odds, and so when wrong are penalized infinitely — we applied a cutoff to extreme probability estimates supported by MML theory Dowe, 2000, enforcing the range:

$$
\left[\frac{(1 / 2)}{(n+1)}, \frac{(n+1 / 2)}{(n+1)}\right]
$$

where $n$ is the sample size.

Table 1. Predictive accuracy results ( \pm 1.96 standard deviations). Boldface indicates statistically significant differences for high results (relative to non-boldface results).

\begin{tabular}{l|l|l|l|l|r} 
Data set & \multicolumn{1}{|c}{ C4.5 } & \multicolumn{1}{c}{ MML Tree } & \multicolumn{1}{|c|}{ MML Graph } & \multicolumn{1}{|c}{ Naive Bayes } & \multicolumn{1}{c}{ DStump } \\
\hline BC & $0.700 \pm 0.079$ & $0.685 \pm 0.085$ & $0.698 \pm 0.064$ & $0.706 \pm 0.062$ & $0.682 \pm 0.055$ \\
CH & $\mathbf{0 . 9 9 2} \pm \mathbf{0 . 0 0 7}$ & $\mathbf{0 . 9 8 9} \pm \mathbf{0 . 0 0 9}$ & $\mathbf{0 . 9 8 4} \pm \mathbf{0 . 0 1 4}$ & $0.704 \pm 0.062$ & $0.676 \pm 0.020$ \\
GL & $0.663 \pm 0.112$ & $0.623 \pm 0.142$ & $0.607 \pm 0.137$ & $0.626 \pm 0.098$ & $0.558 \pm 0.134$ \\
G2 & $0.772 \pm 0.120$ & $0.742 \pm 0.094$ & $0.737 \pm 0.129$ & $0.780 \pm 0.139$ & $0.711 \pm 0.110$ \\
HD & $0.759 \pm 0.086$ & $0.615 \pm 0.083$ & $0.628 \pm 0.060$ & $0.808 \pm 0.084$ & $0.732 \pm 0.059$ \\
HE & $0.802 \pm 0.135$ & $0.796 \pm 0.072$ & $0.782 \pm 0.060$ & $0.809 \pm 0.097$ & $0.812 \pm 0.091$ \\
HO & $0.858 \pm 0.052$ & $0.857 \pm 0.064$ & $0.853 \pm 0.063$ & $0.807 \pm 0.070$ & $0.817 \pm 0.063$ \\
HY & $\mathbf{0 . 9 9 2} \pm \mathbf{0 . 0 0 6}$ & $\mathbf{0 . 9 9 3} \pm \mathbf{0 . 0 0 4}$ & $\mathbf{0 . 9 9 2} \pm \mathbf{0 . 0 0 5}$ & $0.951 \pm 0.007$ & $0.972 \pm 0.006$ \\
IR & $0.935 \pm 0.065$ & $0.942 \pm 0.049$ & $0.943 \pm 0.065$ & $0.896 \pm 0.089$ & $0.875 \pm 0.075$ \\
LA & $0.773 \pm 0.226$ & $0.629 \pm 0.170$ & $0.663 \pm 0.193$ & $0.848 \pm 0.150$ & $0.796 \pm 0.120$ \\
LY & $0.781 \pm 0.089$ & $0.718 \pm 0.137$ & $0.691 \pm 0.109$ & $0.782 \pm 0.119$ & $0.733 \pm 0.113$ \\
MU & $\mathbf{1 . 0 0 0} \pm \mathbf{0 . 0 0 0}$ & $\mathbf{1 . 0 0 0} \pm \mathbf{0 . 0 0 0}$ & $\mathbf{1 . 0 0 0} \pm \mathbf{0 . 0 0 0}$ & $0.910 \pm 0.010$ & $0.986 \pm 0.004$ \\
SE & $\mathbf{0 . 9 7 8} \pm \mathbf{0 . 0 0 7}$ & $\mathbf{0 . 9 7 8} \pm \mathbf{0 . 0 0 8}$ & $\mathbf{0 . 9 7 7} \pm \mathbf{0 . 0 0 9}$ & $0.909 \pm 0.014$ & $0.902 \pm 0.015$ \\
SO & $0.975 \pm 0.087$ & $0.988 \pm 0.050$ & $0.975 \pm 0.128$ & $0.960 \pm 0.212$ & $0.777 \pm 0.166$ \\
VO & $\mathbf{0 . 9 6 1} \pm \mathbf{0 . 0 3 0}$ & $\mathbf{0 . 9 5 4} \pm \mathbf{0 . 0 2 5}$ & $\mathbf{0 . 9 5 3} \pm \mathbf{0 . 0 3 6}$ & $0.889 \pm 0.037$ & $\mathbf{0 . 9 5 6} \pm \mathbf{0 . 0 2 2}$ \\
V1 & $0.890 \pm 0.049$ & $0.881 \pm 0.040$ & $0.871 \pm 0.044$ & $0.869 \pm 0.052$ & $0.874 \pm 0.053$
\end{tabular}

In general, information reward follows predictive accuracy fairly closely. Since information reward is a partial function of accuracy, this is unsurprising. But we would expect information reward to diverge significantly from the assessment of predictive accuracy when the calibration differs substantially between learners. And, in these results we see exactly that in a number of cases. For example, C4.5 performs well in predictive accuracy on the glass data (GL; Table 1); however, its information reward for GL is clearly the worst (despite high variance; Table 2), which is explained by its high miscalibration rating.

The pattern of correlations between the three variables is striking: predictive accuracy is fairly strongly negatively correlated with miscalibration (Table 6); predictive accuracy is even more strongly positively correlated with information reward (Table 4); and miscalibration is negatively correlated with information reward (Table 5). This pattern is consistent with our interpretation above of information reward. 
Table 2. Average information reward ( \pm 1.96 standard deviations). Boldface indicates statistically significant differences for high results (relative to non-boldface results).

\begin{tabular}{l|r|r|r|r|r} 
Data set & \multicolumn{1}{|c}{ C4.5 } & \multicolumn{1}{c}{ MML Tree } & MML Graph & Naive Bayes & \multicolumn{1}{c}{ DStump } \\
\hline BC & $0.016 \pm 0.269$ & $0.131 \pm 0.173$ & $0.157 \pm 0.098$ & $0.136 \pm 0.194$ & $0.095 \pm 0.167$ \\
CH & $\mathbf{0 . 9 4 5} \pm \mathbf{0 . 0 5 1}$ & $\mathbf{0 . 9 3 0} \pm \mathbf{0 . 0 4 4}$ & $\mathbf{0 . 9 4 6} \pm \mathbf{0 . 0 3 5}$ & $0.200 \pm 0.036$ & $0.097 \pm 0.025$ \\
GL & $-0.809 \pm 0.859$ & $0.034 \pm 0.181$ & $0.018 \pm 0.174$ & $0.147 \pm 0.086$ & $-0.058 \pm 0.214$ \\
G2 & $-0.206 \pm 0.827$ & $0.096 \pm 0.343$ & $0.128 \pm 0.261$ & $0.321 \pm 0.194$ & $-0.095 \pm 0.401$ \\
HD & $-0.215 \pm 0.457$ & $0.059 \pm 0.088$ & $0.062 \pm 0.077$ & $0.409 \pm 0.171$ & $0.122 \pm 0.127$ \\
HE & $0.180 \pm 0.452$ & $0.307 \pm 0.152$ & $0.283 \pm 0.184$ & $0.297 \pm 0.412$ & $0.244 \pm 0.341$ \\
HO & $0.374 \pm 0.209$ & $0.403 \pm 0.160$ & $0.403 \pm 0.134$ & $0.325 \pm 0.178$ & $0.329 \pm 0.151$ \\
HY & $\mathbf{0 . 9 3 3} \pm \mathbf{0 . 0 6 0}$ & $\mathbf{0 . 9 4 8} \pm \mathbf{0 . 0 2 7}$ & $\mathbf{0 . 9 4 2} \pm \mathbf{0 . 0 3 1}$ & $0.526 \pm 0.084$ & $\mathbf{0 . 8 9 0} \pm \mathbf{0 . 0 6 0}$ \\
IR & $0.604 \pm 0.443$ & $0.678 \pm 0.213$ & $0.659 \pm 0.352$ & $0.378 \pm 0.090$ & $0.593 \pm 0.165$ \\
LA & $0.086 \pm 0.543$ & $0.020 \pm 0.201$ & $0.042 \pm 0.157$ & $0.591 \pm 0.395$ & $0.185 \pm 0.569$ \\
LY & $-0.047 \pm 0.485$ & $0.132 \pm 0.188$ & $0.092 \pm 0.144$ & $0.331 \pm 0.229$ & $0.230 \pm 0.208$ \\
MU & $\mathbf{1 . 0 0 0} \pm \mathbf{0 . 0 0 0}$ & $\mathbf{1 . 0 0 0} \pm \mathbf{0 . 0 0 0}$ & $\mathbf{0 . 9 9 9} \pm \mathbf{0 . 0 1 1}$ & $0.636 \pm 0.038$ & $0.908 \pm 0.018$ \\
SE & $\mathbf{0 . 8 4 9} \pm \mathbf{0 . 0 5 1}$ & $\mathbf{0 . 8 7 4} \pm \mathbf{0 . 0 3 7}$ & $\mathbf{0 . 8 6 8} \pm \mathbf{0 . 0 3 1}$ & $0.025 \pm 0.174$ & $\mathbf{0 . 5 8 6} \pm \mathbf{0 . 2 3 7}$ \\
SO & $0.828 \pm 0.518$ & $0.903 \pm 0.299$ & $0.865 \pm 0.448$ & $0.721 \pm 0.315$ & $0.496 \pm 0.339$ \\
VO & $0.809 \pm 0.106$ & $0.784 \pm 0.097$ & $0.766 \pm 0.159$ & $0.587 \pm 0.136$ & $0.764 \pm 0.116$ \\
V1 & $0.528 \pm 0.226$ & $0.536 \pm 0.134$ & $0.530 \pm 0.117$ & $0.518 \pm 0.173$ & $0.459 \pm 0.167$
\end{tabular}

Table 3. Miscalibration results ( \pm 1.96 standard deviations). Boldface indicates statistically significant differences for high results (relative to non-boldface results)

\begin{tabular}{l|c|r|r|r|r} 
Data set & C4.5 & MML Tree & MML Graph & Naive Bayes & \multicolumn{1}{c}{ DStump } \\
\hline BC & $0.562 \pm 0.277$ & $0.516 \pm 0.274$ & $0.475 \pm 0.232$ & $0.571 \pm 0.276$ & $\mathbf{1 . 1 7 6} \pm \mathbf{0 . 2 3 9}$ \\
CH & $0.309 \pm 0.215$ & $0.383 \pm 0.159$ & $0.411 \pm 0.299$ & $2.155 \pm 0.236$ & $\mathbf{4 . 9 2 5} \pm \mathbf{0 . 0 9 9}$ \\
GL & $0.855 \pm 0.281$ & $0.472 \pm 0.279$ & $0.505 \pm 0.302$ & $0.437 \pm 0.245$ & $0.678 \pm 0.284$ \\
G2 & $0.461 \pm 0.299$ & $0.363 \pm 0.266$ & $0.359 \pm 0.311$ & $0.312 \pm 0.170$ & $0.451 \pm 0.248$ \\
HD & $0.717 \pm 0.326$ & $0.549 \pm 0.244$ & $0.512 \pm 0.206$ & $0.412 \pm 0.204$ & $0.501 \pm 0.176$ \\
HE & $0.412 \pm 0.258$ & $0.290 \pm 0.161$ & $0.299 \pm 0.156$ & $0.471 \pm 0.348$ & $0.355 \pm 0.204$ \\
HO & $0.446 \pm 0.149$ & $0.432 \pm 0.165$ & $0.428 \pm 0.207$ & $0.462 \pm 0.211$ & $0.497 \pm 0.133$ \\
HY & $0.326 \pm 0.244$ & $0.301 \pm 0.134$ & $0.317 \pm 0.119$ & $\mathbf{1 . 7 3 2} \pm \mathbf{0 . 2 6 6}$ & $0.789 \pm 0.271$ \\
IR & $0.204 \pm 0.184$ & $0.192 \pm 0.114$ & $0.187 \pm 0.140$ & $\mathbf{0 . 5 7 2} \pm \mathbf{0 . 1 9 0}$ & $\mathbf{0 . 5 3 1} \pm \mathbf{0 . 3 0 2}$ \\
LA & $0.157 \pm 0.159$ & $0.093 \pm 0.183$ & $0.124 \pm 0.166$ & $0.155 \pm 0.059$ & $0.157 \pm 0.111$ \\
LY & $0.489 \pm 0.219$ & $0.392 \pm 0.232$ & $0.368 \pm 0.183$ & $0.329 \pm 0.185$ & $0.354 \pm 0.167$ \\
MU & $0.000 \pm 0.000$ & $0.002 \pm 0.002$ & $0.013 \pm 0.116$ & $\mathbf{2 . 3 8 6} \pm \mathbf{0 . 3 1 2}$ & $1.070 \pm 0.223$ \\
SE & $0.551 \pm 0.165$ & $0.528 \pm 0.147$ & $0.515 \pm 0.190$ & $\mathbf{1 . 8 3 5} \pm \mathbf{0 . 2 5 2}$ & $\mathbf{1 . 8 8 1} \pm \mathbf{0 . 5 1 4}$ \\
SO & $0.026 \pm 0.089$ & $0.016 \pm 0.043$ & $0.026 \pm 0.103$ & $0.181 \pm 0.102$ & $0.213 \pm 0.089$ \\
VO & $0.262 \pm 0.177$ & $0.284 \pm 0.201$ & $0.321 \pm 0.165$ & $0.507 \pm 0.229$ & $0.268 \pm 0.118$ \\
V1 & $0.408 \pm 0.227$ & $0.448 \pm 0.200$ & $0.427 \pm 0.185$ & $0.515 \pm 0.277$ & $0.459 \pm 0.199$
\end{tabular}

The results of testing information reward are unlikely to revolutionize data mining practice. However, they can tell an importantly different story from that of accuracy measures alone. For example, both Naive Bayes and decision stumps have received much publicity in recent years as techniques which are effective on many problems while being supremely simple. The results here show that they are both much more poorly calibrated than the more sophisticated methods they might replace and so that they are worse performers than many had thought. 
Table 4. Correlations between information reward and predictive accuracy. ( $N a N$ (not a number) occurs because one of the datasets for correlation consists entirely of 1's.)

\begin{tabular}{l|r|r|r|r|r} 
Data set & C4.5 & MML Tree & MML Graph & Naive Bayes & DStump \\
\hline BC & 0.410 & 0.810 & 0.689 & 0.865 & 0.434 \\
CH & 0.902 & 0.834 & 0.703 & 0.960 & 0.930 \\
GL & 0.711 & 0.388 & 0.558 & 0.499 & 0.308 \\
G2 & 0.823 & 0.594 & 0.868 & 0.960 & 0.477 \\
HD & 0.659 & 0.868 & 0.554 & 0.910 & 0.893 \\
HE & 0.879 & 0.605 & 0.689 & 0.901 & 0.648 \\
HO & 0.855 & 0.928 & 0.905 & 0.878 & 0.899 \\
HY & 0.939 & 0.867 & 0.872 & 0.988 & 0.638 \\
IR & 0.955 & 0.879 & 0.916 & 0.518 & 0.957 \\
LA & 0.725 & 0.928 & 0.833 & 0.880 & 0.538 \\
LY & 0.658 & 0.789 & 0.730 & 0.817 & 0.715 \\
MU & $N a N$ & $N a N$ & 1.000 & 0.881 & 0.998 \\
SE & 0.756 & 0.916 & 0.822 & 0.990 & 0.226 \\
SO & 1.000 & 1.000 & 0.948 & 0.937 & 0.803 \\
VO & 0.791 & 0.772 & 0.766 & 0.746 & 0.905 \\
V1 & 0.814 & 0.603 & 0.708 & 0.895 & 0.787
\end{tabular}

Table 5. Correlations between information reward and miscalibration.

\begin{tabular}{l|r|r|r|r|r} 
Data set & C4.5 & MML Tree & MML Graph & Naive Bayes & DStump \\
\hline BC & -0.602 & -0.664 & -0.087 & -0.762 & 0.231 \\
CH & -0.800 & -0.417 & -0.701 & 0.405 & -0.893 \\
GL & -0.892 & -0.788 & -0.736 & 0.398 & -0.492 \\
G2 & -0.932 & -0.712 & -0.734 & -0.253 & -0.610 \\
HD & -0.827 & -0.479 & -0.152 & -0.546 & -0.261 \\
HE & -0.725 & -0.191 & -0.266 & -0.833 & -0.646 \\
HO & -0.487 & -0.565 & -0.520 & -0.462 & -0.384 \\
HY & -0.855 & -0.648 & -0.394 & -0.559 & 0.201 \\
IR & -0.929 & -0.634 & -0.761 & 0.052 & -0.988 \\
LA & -0.750 & -0.667 & -0.198 & -0.774 & -0.468 \\
LY & -0.746 & -0.239 & -0.285 & -0.046 & -0.244 \\
MU & $N a N$ & -0.639 & -0.999 & -0.755 & -0.914 \\
SE & -0.591 & -0.631 & -0.575 & -0.781 & 0.638 \\
SO & -1.000 & -0.998 & -0.957 & -0.851 & -0.702 \\
VO & -0.631 & -0.110 & -0.447 & -0.696 & -0.347 \\
V1 & -0.616 & -0.297 & -0.567 & -0.680 & -0.635
\end{tabular}


Table 6. Correlations between predictive accuracy and miscalibration.

\begin{tabular}{l|r|r|r|r|r} 
Data set & C4.5 & MML Tree & MML Graph & Naive Bayes & DStump \\
\hline BC & 0.606 & -0.631 & -0.326 & -0.878 & -0.330 \\
CH & -0.940 & -0.455 & -0.752 & 0.491 & -0.838 \\
GL & -0.829 & -0.588 & -0.551 & 0.293 & -0.035 \\
G2 & -0.838 & -0.545 & -0.562 & -0.344 & -0.692 \\
HD & -0.834 & -0.519 & -0.344 & -0.447 & -0.269 \\
HE & -0.710 & 0.412 & 0.016 & -0.946 & -0.647 \\
HO & -0.235 & -0.497 & -0.499 & -0.287 & -0.228 \\
HY & -0.805 & -0.488 & -0.495 & -0.622 & 0.086 \\
IR & -0.882 & -0.400 & -0.703 & 0.834 & -0.955 \\
LA & -0.797 & -0.351 & 0.345 & -0.817 & -0.518 \\
LY & -0.461 & -0.127 & -0.211 & 0.107 & -0.102 \\
MU & $N a N$ & $N a N$ & -1.000 & -0.682 & -0.911 \\
SE & -0.642 & -0.598 & -0.741 & -0.811 & -0.005 \\
SO & -1.000 & -0.998 & -0.999 & -0.644 & -0.815 \\
VO & -0.807 & 0.419 & -0.581 & -0.656 & -0.397 \\
V1 & -0.789 & -0.350 & -0.534 & -0.866 & -0.738
\end{tabular}

Table 7. Properties of the datasets employed.

\begin{tabular}{l|r|r|r|r} 
Dataset & Training set size & Test set size & Attributes & Classes \\
\hline BC & 190 & 96 & 9 & 2 \\
CH & 2130 & 1066 & 36 & 2 \\
GL & 142 & 72 & 9 & 6 \\
G2 & 108 & 55 & 9 & 2 \\
HD & 202 & 101 & 13 & 2 \\
HE & 103 & 52 & 19 & 2 \\
HO & 245 & 123 & 22 & 2 \\
HY & 2108 & 1055 & 25 & 2 \\
IR & 100 & 50 & 4 & 3 \\
LA & 38 & 19 & 16 & 2 \\
LY & 98 & 50 & 18 & 4 \\
MU & 5416 & 2708 & 22 & 2 \\
SE & 2108 & 1055 & 25 & 2 \\
SO & 31 & 16 & 35 & 4 \\
VO & 290 & 145 & 16 & 2 \\
V1 & 290 & 145 & 15 & 2
\end{tabular}




\section{Conclusion}

Information reward provides an effective, general-purpose performance measure for machine learning algorithms. It combines measures of domain understanding and calibration, and it provides means for these two to be analyzed separately and together. In future work we will compare information reward with ROC and lift curves, which provide another popular evaluation method. We will also look at using feedback on calibration (over- and under-confidence) to improve performance in incremental learning tasks.

Acknowledgement. We acknowledge the Monash Data Mining Centre for assisting with the experimental work reported here. Thanks to Richard McConachy for useful comments.

\section{References}

[Brier, 1950] Brier, G.W. (1950). Verification of forecasts expressed in terms of probability. Monthly Weather Review, 78, 1-3.

[Cover and Thomas, 1991] Cover, T.M. and Thomas, J.A. (1991). Elements of information theory. New York: Wiley.

[Domingos and Pazzani 1996] Domingos, P. and Pazzani, M. (1996). Beyond independence: Conditions for the optimality of the simple bayesian classifier. In Proceedings of the Thirteenth International Conference on Machine Learning, (pp. 105-112), Bari, Italy. Morgan Kaufmann.

[Dowe et al., 1996] Dowe, D.L., Farr, G.E., Hurst, A.J. and Lentin, K.L. (1996). Information-theoretic football tipping. Technical Report 96/297, Dept. of Computer Science, Monash University.

[Dowe, 2000] Dowe, D.L. (2000). Learning and prediction notes. School of Computer Science and Software Engineering, Monash University.

[Good, 1952] Good, I.J. (1952). Rational decisions. Jrn. of the Royal Statistical Society, B, 14, 107-114. Reprinted in Good thinking: The foundations of probability and its applications, Minnesota, 1983.

[Griffen and Tversky, 1992] Griffen, D., and Tversky, A. (1992). The weighing of evidence and the determinants of confidence. Cognitive Psychology, 24, 411-435.

[Holte, 1993] Holte, R. C., (1993). Very simple classification rules perform well on most commonly used datasets. Machine Learning, 11, 63-91.

[Jeffrey, 1983] Jeffrey, R. (1983). The logic of decision, 2nd ed. New York: McGrawHill.

[Kononenko and Bratko, 1991] Kononenko, I., and Bratko, I. (1991). Informationbased evaluation criterion for classifier's performance. Machine Learning, 6, 67-80.

[Leslie, 1998] Leslie, C. (1998). Lack of confidence. MA Thesis, Department of History and Philosophy of Science, University of Melbourne.

[Lewis, 1980] Lewis, D. (1980). A subjectivist's guide to objective chance. In Jeffrey (Ed.) Studies in inductive logic and probability, vol II (pp. 263-293). Univ of California.

[Lichtenstein et al., 1977] Lichtenstein, S., Fischhoff, B., and Phillips, L.D. (1977). Calibration of probabilities: The state of the art. In H. Jungermann and G. de Zeeuw (Eds.), Decision making and change in human affairs (pp. 275-324). Dordrecht: Reidel. 
[Matheson and Winkler, 1976] Matheson, J.E., and Winkler, R. L. (1976). Scoring rules for continuous probability distributions. Management Science, 22.

[McClelland and Bolger, 1994] McClelland, A. G. R., and Bolger, F. (1994). The calibration of subjective probabilities: Theories and models, 1980-1994. In G. Wright and P. Ayton (Eds.) Subjective probability, Wiley.

[Mitchell, 1997] Mitchell, T. (1997]). Machine learning. McGraw-Hill.

[Morgan and Henrion, 1990] Morgan, M. G., and Henrion, M. (1990). Uncertainty: A guide to dealing with uncertainty in quantitative risk and policy analysis. Cambridge University.

[Murphy and Winkler, 1984] Murphy, A. H., and Winkler, R. L. (1984). The probability of precipitation forecasts. Journal of the American Statistical Association, 79, 391-400.

[Oliver, 1993] Oliver, J. (1993). Decision graphs - an extension of decision trees. Fourth Int. Conf. Artificial Intelligence and Statistics, pp. 343-350

[Pearl, 1978] Pearl, J. (1978). An economic basis for certain methods of evaluating probabilistic forecasts. International Journal of Man-Machine Studies, 10, 175183.

[Provost et al., 1998] Provost, F., Fawcett, T. and Kohavi, R. (1998). The case against accuracy estimation for comparing induction algorithms. International Conference on Machine Learning, 1998, Morgan Kaufmann.

[Quinlan, 1993] Quinlan, J. R. (1993). C4.5: programs for machine learning. Morgan Kaufmann.

[Ramsey, 1931] Ramsey, F.P. (1931). The foundations of mathematics and other logical essays, edited by R.B. Braithwaite. New York: Humanities Press.

[Savage, 1971] Savage, L.J. (1971). Elicitation of personal probabilities and expectations. Journal of the American Statistical Association, 66.

[Turney, 1995] Turney, P. (1995). Cost-sensitive classification: Empirical evaluation of a hybrid genetic decision tree induction algorithm. Journal of Artificial Intelligence Research, 369-409.

[Wallace and Boulton, 1968] Wallace, C.S., and Boulton, D.M. (1968). An information measure for classification. The Computer Journal, 11, 185-194.

[Wallace and Patrick, 1993] Wallace, C.S., and Patrick, J. D. (1993). Coding decision trees. Machine Learning, 11, 7-22. 